\title{
Exercise is delayed in critically ill patients: A five year observational study in an Australian tertiary intensive care unit
}

Marc Nickels* 1, 2, Leanne Aitken 3, 4, 5, James Walsham4, 6, Lisa Watson'1, Steven McPhail 2, 7

${ }^{1}$ Intensive Care Unit and Physiotherapy Department, Princess Alexandra Hospital, ${ }^{2}$ School of Public Health \& Social

Work and Institute of Health and Biomedical Innovation, Queensland University of Technology, ${ }^{3}$ NHMRC Centre of Research Excellence in Nursing Interventions for Hospitalised Patients, Menzies Health Institute Queensland, Griffith University , ${ }^{4}$ Intensive Care Unit, Princess Alexandra Hospital, Brisbane, Australia, ${ }^{5}$ School of Health Sciences, City University London, London, United Kingdom, ${ }^{6}$ School of Medicine, University Of Queensland, ${ }^{7}$ Centre for Functioning and Health Research, Metro South Health, Brisbane, Australia

Duration of bed rest among critically ill patients in ICU has been associated with development of persistent weakness that can last for more than five years. Commencing early exercise interventions in ICU is likely to reduce critically ill patients' physical dysfunction. However, critically ill patients often experience prolonged periods of bed rest and inactivity.

This study examined the timing of commencement of exercise interventions, including sitting out of bed and upright mobilisation, following physiological stability in critically ill patients and describes key clinical outcomes.

Participants included consecutive patients admitted for $>48$ hours to a 25-bed Australian mixed medical and surgical adult ICU between July 2009 and June 2014. Time taken for patients to achieve neurological, cardiorespiratory and cardiovascular (physiological) stability was calculated and timing of initial sitting out of bed and upright mobilisation was recorded.

A small number of patients $(\mathrm{n}=206,6.0 \%)$ did not achieve physiological stability. A substantial proportion of patients $(\mathrm{n}=1377,40.1 \%)$ did not complete any mobilisation or sitting activities. For patients $(\mathrm{n}=1851,53.9 \%)$ who did undertake mobilisation or sitting activities, activity commenced a median (IQR) of $3.6(2.0,7.7)$ days after ICU admission. This represented a median (IQR) delay after physiological stability of $2.3(1.3,4.4)$ days for mobilisation and $2.7(1.5,5.7)$ days for sitting. In-hospital mortality was $14.3 \%(\mathrm{n}=491)$ for patients who did not participate in exercise interventions, compared to $2.6 \%(\mathrm{n}=89)$ for patients who exercised whilst in ICU.

Despite known benefits of early exercise with critically ill patients, many stable patients did not commence any exercise in ICU, or the commencement of exercise was somewhat delayed. This study indicates there may be opportunity to further improve patient outcomes through timely implementation of exercise-based interventions.

Disclosure of Interest: None Declared 